\title{
Is there a relationship between surgical success defined by motor and health-related quality of life criteria in adult strabismic patients?
}

\author{
Peng Yong Sim $\mathbb{1}^{1} \cdot$ Saurabh Jain ${ }^{1,2}$ \\ Received: 13 November 2017 / Accepted: 1 December 2017 / Published online: 5 February 2018 \\ (c) The Royal College of Ophthalmologists 2018
}

Different outcome criteria (namely motor, diplopia and health-related quality of life (HRQOL)) currently exist to evaluate postoperative success following adult strabismus surgery [1]. Of these, motor alignment is the most frequently applied criterion in the literature to define a desirable outcome [2]. However, this falls short of addressing other key aspects such as the impact of strabismus surgery on patients' quality of life. Correspondingly, the HRQOL criterion has gained increasing popularity due to its utility in providing a holistic assessment of surgical outcomes [3].

While the success rates of different criteria have been described [1], there is currently no literature exploring the relationship between the success rates in these criteria. We therefore evaluated success using motor and HRQOL criteria in adult strabismic patients and investigated any relationship between the two.

Case notes of 87 consecutive adult patients undergoing strabismus surgery were analysed. For motor criterion, success was defined as $<10$ prism dioptres (PD) of orthotropia postoperatively. For HRQOL criterion, this was assessed using the strabismus-specific AS-20 questionnaire and success was defined as a preoperative to postoperative change in AS-20 score exceeding previously published $95 \%$ limits of agreement [4].

Eighty-seven patients (mean age 47 years; $53 \%$ female) were included. The median preoperative deviation and AS20 score were $30 \mathrm{PD}(95 \%$ confidence interval $(\mathrm{CI}) \pm 3.9)$ and 58.8 (95\% CI \pm 4.6$)$, respectively. Postoperatively, the median deviation and AS-20 score were 10.2 PD (95\% CI \pm $2.3)$ and 72.5 (95\% CI \pm 4.4$)$, respectively. There was a

\footnotetext{
$\triangle$ Peng Yong Sim

pengyong91@gmail.com

1 Royal Free Hospital, London, UK

2 University College London Medical School, London, UK
}

significant improvement in both measures postoperatively $(p<0.001)$. When applying motor and HRQOL criteria, $74 \%(64 / 87)$ and $62 \%$ (54/87) of surgeries were classified as success, respectively. Statistical analysis showed no correlation between surgical success defined by motor and HRQOL criteria (Phi's coefficient $-0.039, p=0.72$ ).

Our success rates for motor and HRQOL criteria compares favourably with existing literature [1, 5]. Although we observed an overall significant improvement in patients' deviation and HRQOL after strabismus surgery, we found no association between the success rates defined by the two criteria. This suggests that application of a single criterion alone to define success might be of limited use, and that incorporation of different criteria may provide a more clinically representative assessment of outcome in strabismus surgery.

\section{Compliance with ethical standards}

Conflict of interest The authors declare that they have no competing interests.

\section{References}

1. Hatt SR, Leske DA, Bradley EA, et al. Comparison of quality of life instruments in adults with strabismus. Am J Ophthalmol. 2009; 148:558-62.

2. Mills MD, Coats DK, Doanhue SP, et al. Strabismus surgery for adults: a report by the American Academy of Ophthalmology. Ophthalmology. 2004;111:1255-62.

3. Hatt SR, Leske DA, Bradley EA, et al. Development of a Qualityof-Life Questionnaire for adults with strabismus. Ophthalmology. 2009;116:1-12.

4. Leske DA, Hatt SR, Holmes JM. Test-retest reliability of HealthRelated Quality-of-Life Questionnaires in adults with strabismus. Am J Ophthalmol. 2010;149:672-6.

5. Keech RV, Scott WE, Christensen LE. Adjustable suture strabismus surgery. J Pediatr Ophthalmol Strabismus. 1998;35:138-45. 